\title{
Silent cerebral microbleeds associated with arterial stiffness in an apparently healthy subject
}

\author{
Namiko Ochi ${ }^{1}$, Yasuharu Tabara ${ }^{2}$, Michiya Igase $^{1}$, Tokihisa Nagai ${ }^{1}$, Tomoko Kido ${ }^{1}$, Tetsuro Miki ${ }^{1}$ \\ and Katsuhiko Kohara ${ }^{1}$
}

Silent cerebral microbleeds (MBs) are a common finding in stroke patients, especially those with intracerebral hemorrhage, and are thought to be a marker of future cerebral hemorrhage. Clinically, two distinct forms of MBs have been documented, those observed with either or both stroke or small vessel disease (SVD) and those associated with cerebral amyloid angiopathy. We investigated a possible association between MBs and arterial stiffness in a general population. Subjects were $\mathbf{4 4 3}$ apparently healthy individuals with a mean age of $67.1 \pm 8.1$ years. The presence of MBs, lacunar infarcts and periventricular hyperintensity (PVH) was determined by 3-tesla magnetic resonance imaging. Carotid intima-media thickness (IMT) was measured by ultrasonography. Arterial stiffness was evaluated by brachial-to-ankle pulse wave velocity (baPWV), and the Framingham stroke risk score (FSRS) was obtained as an integrated cerebrovascular risk factor. The prevalence of MBs was 5.0\%. Both baPWV and FSRS were significantly higher in subjects with MBs ( $1820 \pm 308$ vs. $1645 \pm 325 \mathrm{~cm} / \mathrm{s}, P=0.014$ and $12.1 \pm 8.6$ vs. $8.9 \pm 7.5 \%, P=0.047$, respectively). Odds ratio of a high baPWV, defined as $\geqslant 1500 \mathrm{~cm} / \mathrm{s}$, for the presence of MBs was 6.05 even after correction for confounding parameters, including age and hypertension. This association with high baPWV remained irrespective of MBs location, whether strictly located in the lobes or in the basal ganglia and infratentorial regions. These findings indicate an association between arterial stiffness and the presence of MBs. Assessment of arterial stiffness may be useful in identifying subjects at high risk for the presence of MBs. Hypertension Research (2009) 32, 255-260; doi:10.1038/hr.2009.13; published online 27 February 2009

Keywords: arterial stiffness; cerebral amyloid angiopathy; microbleeds; pulse wave velocity; small vessel disease

\section{INTRODUCTION}

The term microbleeds (MBs) was coined by Offenbacher et al. ${ }^{1}$ to describe small hypointense lesions on gradient echo ( $\mathrm{T} 2^{\star}$-weighted) MRI. The wide clinical availability of this equipment has attracted much interest in asymptomatic cerebral MBs, with findings to date showing a frequent occurrence in patients with stroke, particularly those with intracerebral hemorrhage, ${ }^{2-4}$ higher prevalence in patients with recurrent intracerebral hemorrhage than in those with a first episode $;^{5-7}$ and that their presence predicts bleeding after thrombolytic therapy ${ }^{8}$ and antiplatelet drug administration in ischemic stroke. ${ }^{9}$ These findings have in turn raised concerns that MBs may be a marker for future symptomatic cerebral hemorrhage. Interestingly, although MBs are also observed in apparently healthy subjects, albeit at a far lower prevalence, ${ }^{2-4}$ their pathological significance in these subjects remains to be determined.

Clinically, two distinct forms of MBs have been documented. ${ }^{2-4,10}$ Those observed with either or both stroke or small vessel disease (SVD), including lacunar infarcts and advanced deep white matter lesions, are frequently located in the basal ganglia and infratentorial regions and are associated with risk factors for stroke. ${ }^{2-4,10}$ In contrast, those located solely in the lobar regions have shown no association with SVD or stroke risk, ${ }^{10}$ and are thought to have cerebral amyloid angiopathy (CAA) as an underlying mechanism..$^{2-4,10,11}$

It is interesting to note that recent findings have also identified an association between MBs and Alzheimer disease (AD). ${ }^{2-4}$ This is conceivable given that MBs are associated with hypertension and CAA, which are both in turn associated with $\mathrm{AD}$ and that the amyloid deposits histologically characteristic of $\mathrm{AD}$ are frequently attributable to previous MBs. ${ }^{12}$ The central role of vascular factors in not only vascular dementia but also $\mathrm{AD}$ has been recognized; in particular, pulse wave velocity (PWV), an index of arterial stiffness, has been related with cognitive impairments, including $\mathrm{AD},{ }^{13}$ whereas $\mathrm{SVD}$, including MBs, are speculated to result from abnormal flow pulsations extending into the small cerebral vessels as a consequence of aortic stiffening. ${ }^{14}$ These various findings indicate the possible association of MBs with arterial stiffness.

Here, we investigated the hypothesis that arterial stiffness is associated with MBs in a population of apparently healthy subjects. We also used two parameters representing different aspects of atherosclerosis, carotid IMT and baPWV, to assess whether parameters for atherosclerosis might be used to improve the prediction of MBs in a general population. Finally, we evaluated the integration of known risk factors for stroke with the Framingham stroke risk score (FSRS).

${ }^{1}$ Department of Geriatric Medicine, Ehime University Graduate School of Medicine, Ehime, Japan and ${ }^{2}$ Department of Basic Medical Research and Education, Ehime University Graduate School of Medicine, Ehime, Japan

Correspondence: Dr K Kohara, Department of Geriatric Medicine, Ehime University Graduate School of Medicine, Shitsukawa, Toon City, Ehime 791-0295, Japan.

E-mail: koharak@m.ehime-u.ac.jp

Received 31 July 2008; revised 29 November 2008; accepted 24 December 2008; published online 27 February 2009 


\section{METHODS}

\section{Study subjects}

Subjects were recruited from consecutive visitors to the Anti-Aging Center at Ehime University Hospital from February 2006 to December 2007. They attended the medical check-up program 'Anti-Aging Doc' which is specifically designed to evaluate aging-related disorders, including atherosclerosis, cardiovascular disease, physical function and mild cognitive impairment. This program is provided to general residents of Ehime Prefecture, Japan, and participants undertook, voluntarily, medical checkups. Among 655 visitors, 443 who agreed with the study aims and protocols and gave written consent to all procedures and who were free of any history of symptomatic cerebrovascular events including TIA, coronary heart disease and congestive heart failure were analyzed. All participants were physically independent and free from severe cognitive dysfunction and dementia. The series of studies were approved by the ethics committee of Ehime University Graduate School of Medicine.

Brain MRI assessment of asymptomatic cerebrovascular damage MRI examination was performed at Sadamoto Hospital 2-3 weeks prior to the medical check-up at Anti-Aging Center. Brain MRI was performed with a 3-tesla scanner (Signa Excite 3.0T; GE Healthcare, Milwaukee, WI, USA) to evaluate asymptomatic cerebrovascular damage including MBs, lacunar infarcts and periventricular hyperintensity (PVH) lesions. T1-weighted axial images (TR $2000.0 \mathrm{~ms}$, TE $16.0 \mathrm{~ms}$, thickness $6.0 \mathrm{~mm}$, gap $1.0 \mathrm{~mm}$, matrix $288 \times 224$ ), T2weighted axial images (TR $4800.0 \mathrm{~ms}$, TE $92.0 \mathrm{~ms}$, thickness $6.0 \mathrm{~mm}$, gap $1.0 \mathrm{~mm}$, matrix $512 \times 256$ ), fluid-attenuated inversion recovery (FLAIR) images (TR $11000.0 \mathrm{~ms}$, TE $140.0 \mathrm{~ms}$, thickness $6.0 \mathrm{~mm}$, gap $1.0 \mathrm{~mm}$, matrix $288 \times 224$ ), and gradient echo (T2*-weighted) images (TR $600.0 \mathrm{~ms}$, TE $7.2 \mathrm{~ms}$, thickness $6.0 \mathrm{~mm}$, gap $1.0 \mathrm{~mm}$, matrix $320 \times 192)$ were obtained parallel to the orbitomeatal line. Twenty-two slices were taken at T1-weighted and FLAIR examination and 20 slices at $\mathrm{T} 2$-weighted and $\mathrm{T} 2^{*}$-weighted examination.

MBs were defined as small (2- to 5-mm diameter) hypointense lesions on $\mathrm{T} 2^{*}$-weighted images. ${ }^{1}$ Hypointense lesions within the subarachnoid space and areas of symmetric hypointensity in the globus pallidus on $\mathrm{T} 2^{*}$-weighted images were considered likely to represent adjacent pial blood vessels and calcifications, respectively, and were therefore excluded. Lacunar infarcts were defined as areas of low signal intensity (3- to 15-mm diameter) on T1-weighted images and of high intensity on T2-weighted and FLAIR images. PVHs were defined as white matter hyperintensities depicted on T2-weighted and FLAIR images ${ }^{15,16}$ in contact with the ventricular wall. PVH was further classified into five grades according to the Japanese Braindock Guidelines 2003, ${ }^{17}$ namely grade 0 , absent or only a 'rim'; grade 1, limited lesion-like 'caps'; grade 2, irregular 'halo'; grade 3, irregular margins and extension into the deep white matter; and grade 4, extension into the deep white matter and subcortical portion. Imaging analysis was performed by a neurologist without clinical information on the subject.

\section{Evaluation of carotid atherosclerosis}

Ultrasonography of the common carotid artery (CCA) was performed using an SSD-3500SV (Aloka Co., Ltd., Tokyo, Japan) with a 7.5-MHz probe. After the patient had rested in the supine position for at least $10 \mathrm{~min}$, optimal visualization of the bilateral carotid arteries was obtained with the subject's head tilted slightly upward in the mid-line position. Intima-media thickness (IMT) of the far wall of the CCA was measured using computerized software, which simultaneously measured IMT at three points at $1-\mathrm{cm}$ intervals with three separate cursors optimally positioned on a B-mode image. Nine IMTs of the far wall were measured at three contiguous sites at a $1-\mathrm{cm}$ interval proximal to the bulb from the anterior, lateral and posterior approaches in each side and then averaged to obtain mean IMT. Plaque was defined as the presence of wall thickening of $50 \%$ or greater than the thickness of the surrounding wall, with a minimum absolute thickness of $1.1 \mathrm{~mm} \cdot{ }^{17-19}$ Observation of plaque was performed from $4.5 \mathrm{~cm}$ proximal to $1.5 \mathrm{~cm}$ distal to the bifurcation. Twodimensional guided M-mode tracings of the bilateral CCA at a point $2 \mathrm{~cm}$ proximal to the bulb from the anterior, lateral and posterior approaches were recorded with an electrocardiogram, ${ }^{18}$ and the internal diameters of the CCA at end-diastole (Dd) and peak-systole (Ds) were measured in three cycles and averaged. ${ }^{19}$ Axial resolution of the ultrasonography system was $0.1 \mathrm{~mm}$.

\section{Measurement of brachial-to-ankle pulse wave velocity}

Pulse wave velocity (PWV) was measured using a volume-plethysmographic apparatus (form PWV/ABI; Omron Healthcare Co. Ltd., Kyoto, Japan). A detailed explanation of this device as well as the validity and reproducibility of its measurements have been provided elsewhere. ${ }^{20}$ Briefly, the subject was examined in the supine position, with electrocardiogram electrodes placed on both wrists, a microphone for detecting heart sounds placed on the left edge of the sternum, and cuffs wrapped around a plethysmographic sensor that determined volume pulse waveform and an oscillometric pressure sensor that measures blood pressure. Volume waveforms for the brachium and ankle were stored using a sampling time of $10 \mathrm{~s}$ with automatic gain analysis and quality adjustment. Characteristic points of the waveforms were automatically determined according to the phase velocity theory. ${ }^{20}$

Brachial-to-ankle PWV (baPWV) was calculated from the time interval between the wave fronts of the brachial and ankle waveforms $(\Delta \mathrm{Tba})$ and the path length from the brachium to ankle. Path length from the suprasternal notch to the brachium $(\mathrm{Lb})$ or ankle ( $\mathrm{La}$ ) was obtained using the following formula: $\mathrm{Lb}=0.2195 \times$ height $-2.0734, \mathrm{La}=0.8129 \times$ height +12.328 . baPWV was then obtained as $(\mathrm{La}-\mathrm{Lb}) / \Delta \mathrm{Tba}$.

\section{Risk factor evaluation}

Lifestyle, medical history and prescribed drugs were evaluated by questionnaire. Anthropometric measurements were performed by a trained nurse. Venous blood for measurement of serum lipids and plasma glucose was collected in the morning after at least $12 \mathrm{~h}$ fasting. The homeostasis model assessment index for insulin resistance (HOMA-IR) was calculated as follows: immunoreactive insulin $\left(\mu \mathrm{U} \mathrm{ml}^{-1}\right) \times$ glucose $\left(\mathrm{mg} \mathrm{dl}^{-1}\right) / 405$. Brachial BP was measured using an automatic cuff-oscillometric device (HEM-9000AI; Omron Healthcare Co., Ltd.) after the subject had rested in the sitting position for at least $5 \mathrm{~min}$. Hypertension was defined as any or all of systolic blood pressure (SBP) $\geqslant 140 \mathrm{~mm} \mathrm{Hg}$, diastolic blood pressure (DBP) $\geqslant 90 \mathrm{~mm} \mathrm{Hg}$, or use of an antihypertensive drug. Type 2 diabetes was defined as any or all of fasting plasma glucose $\geqslant 126 \mathrm{mg} \mathrm{dl}^{-1}, \mathrm{HbAlc} \geqslant 6.5 \%$, or use of medication to lower blood glucose levels. Framingham stroke risk score (FSRS) was calculated in each subject as 10-year probability of the first stroke. ${ }^{21}$

\section{Statistical analysis}

All values are expressed as the mean \pm s.d. All statistical analyses were conducted with SAS 8.0.2 (SAS Institute Inc., Cary, NC, USA). Differences between two groups were analyzed by student's $t$-test. Differences in prevalence or frequency were analyzed by the $\chi^{2}$ test. To obtain a baPWV cutoff value, receiver operating characteristic (ROC) curve analysis was performed for the presence of MBs. Factors independently associated with MBs were evaluated using logistic regression analysis. $P$-values less than 0.05 were considered statistically significant.

\section{RESULTS}

\section{Risk factors for MBs}

MBs were identified in 22 of 443 subjects. Three subjects had two MBs and two subjects had three, giving a mean of $1.3 \pm 0.6$. Clinical characteristics of subjects with and without MBs are summarized in Table 1. MBs were associated with older age and hypertension. Although other known risk factors for stroke did not significantly differ between subjects with and without MBs, FSRS and baPWV were significantly higher in those with MBs (Table 1).

Subjects with MBs received antihypertensive agents more frequently than subjects without MBs (Table 1). The distribution of class of antihypertensive drugs is shown in Table 2. ACE inhibitors were more frequently used in hypertensive patients with MBs than those without MBs. $\beta$-blockers were not used in patients with MBs.

\section{Association of small vessel disease and the location of MBs and their relationship with risk factors}

Table 3 shows that MBs were significantly associated with both lacunar infarcts and advanced deep white matter lesions. SVD was defined as 
Table 1 Clinical characteristics of study subjects with microbleeds

\begin{tabular}{|c|c|c|}
\hline & \multicolumn{2}{|c|}{ Microbleeds } \\
\hline & Present $(\mathrm{n}=22)$ & Absent $(\mathrm{n}=421)$ \\
\hline Sex (male/female) & $7 / 15$ & $154 / 268$ \\
\hline Age (years) & $72.2 \pm 5.9 * *$ & $66.8 \pm 8.1$ \\
\hline Body mass index $\left(\mathrm{kg} \mathrm{m}^{-2}\right)$ & $23.3 \pm 3.8$ & $23.0 \pm 3.0$ \\
\hline Systolic blood pressure $(\mathrm{mm} \mathrm{Hg})$ & $146 \pm 23$ & $139 \pm 19$ \\
\hline Diastolic blood pressure (mm Hg) & $81 \pm 13$ & $78 \pm 11$ \\
\hline Pulse pressure $(\mathrm{mm} \mathrm{Hg})$ & $65 \pm 17$ & $60 \pm 14$ \\
\hline LDL cholesterol (mg dl-1) & $123 \pm 27$ & $128 \pm 33$ \\
\hline HDL cholesterol ( $\left.\mathrm{mg} \mathrm{dl}^{-1}\right)$ & $68 \pm 22$ & $68 \pm 19$ \\
\hline Triglyceride $\left(\mathrm{mg} \mathrm{dl}^{-1}\right)$ & $94 \pm 45$ & $106 \pm 59$ \\
\hline Glucose $\left(\mathrm{mg} \mathrm{dl}^{-1}\right)$ & $105 \pm 16$ & $103 \pm 19$ \\
\hline $\mathrm{HbA1c}(\%)$ & $5.6 \pm 0.5$ & $5.6 \pm 0.7$ \\
\hline Immunoreactive insulin $\left(\mu \cup \mathrm{ml}^{-1}\right)$ & $6.1 \pm 3.2$ & $5.9 \pm 4.7$ \\
\hline HOMA-IR & $1.6 \pm 1.0$ & $1.6 \pm 2.0$ \\
\hline Current smokers (\%) & 0 & 6.2 \\
\hline Hypertension (\%) & $90.9^{* *}$ & 54.9 \\
\hline Type 2 diabetes (\%) & 18.2 & 10.2 \\
\hline History of atrial fibrillation (\%) & 4.5 & 0.5 \\
\hline LVH on electrocardiogram (\%) & 4.5 & 11.1 \\
\hline Antihypertensive drugs (\%) & $50.0^{* *}$ & 23.8 \\
\hline Antidiabetic drugs (\%) & 9.1 & 5.2 \\
\hline Antiplatelet drugs (\%) & 4.5 & 4.0 \\
\hline Framingham stroke risk score (\%) & $12.1 \pm 8.6^{*}$ & $8.9 \pm 7.5$ \\
\hline baPWV $\left(\mathrm{cm} \mathrm{s}^{-1}\right)$ & $1820 \pm 308^{*}$ & $1645 \pm 325$ \\
\hline Carotid IMT (mm) & $0.85 \pm 0.14$ & $0.81 \pm 0.15$ \\
\hline Diameter at end diastole $(\mathrm{mm})$ & $6.6 \pm 0.7^{*}$ & $6.3 \pm 0.8$ \\
\hline Diameter at peak systole $(\mathrm{mm})$ & $7.1 \pm 0.8^{*}$ & $6.7 \pm 0.8$ \\
\hline Number of plaques & $1.09 \pm 1.34$ & $0.78 \pm 1.17$ \\
\hline
\end{tabular}

Abbreviations: HDL, high-density lipoprotein; HOMA-IR, Homeostasis model assessment index for insulin resistance; LDL, low-density lipoprotein; LVH, left ventricular hypertrophy. for insulin resistance;

${ }^{*} P<0.05,{ }^{* *} P<0.01$ vs. subjects without lesions.
Values are mean \pm s.d.

Table 2 Antihypertensive drug users in hypertensive patients

\begin{tabular}{lccc}
\hline & \multicolumn{3}{c}{ Microbleeds } \\
\cline { 2 - 4 } & Present $(\mathrm{n}=11) \mathrm{N}(\%)$ & Absent $(\mathrm{n}=100) \mathrm{n}(\%)$ & $\mathrm{P}$ \\
\hline ACE inhibitors & $4(36)$ & $5(5)$ & 0.004 \\
ARBs & $5(41)$ & $41(41)$ & 0.76 \\
CCBs & $7(64)$ & $64(64)$ & 0.98 \\
Diuretics & $2(18)$ & $6(6)$ & 0.19 \\
$\alpha$ blockers & $1(9)$ & $3(3)$ & 0.37 \\
$\beta$ blockers & $0(0)$ & $15(15)$ & 0.06 \\
\hline
\end{tabular}

either or both the presence of lacunar infarcts or PVH $\geqslant 2,50 \%$ of MBs were associated with SVD. Subjects with MBs associated with SVD had significantly higher baPWV than subjects with MBs not associated with SVD $(1952 \pm 341$ vs. $1687 \pm 210 \mathrm{~cm} / \mathrm{s}$, respectively, $P=0.04)$. There were no differences in carotid IMT $(0.90 \pm 0.1 \mathrm{vs}$. $0.80 \pm 0.3 \mathrm{~mm}$, respectively, $P=0.12$ ) and Framingham stroke risk score $(15.4 \pm 10.3$ vs. $8.9 \pm 5.0 \%$, respectively, $P=0.08)$ between two subgroups of MBs.
Table 3 Relationship between cerebral microbleeds and small-vessel disease

\begin{tabular}{llr}
\hline & \multicolumn{2}{c}{ Cerebral microbleeds } \\
\cline { 2 - 3 } & Present $(\mathrm{n}=22)$ & Absent $(\mathrm{n}=421)$ \\
\hline Lacunar infarction, $n(\%)$ & $9(40.9)^{*}$ & $40(9.5)$ \\
Periventricular hyperintensity & & \\
Grade $0(\%)$ & $9(40.9)$ & $241(57.2)$ \\
Grade $1(\%)$ & $6(27.3)$ & $153(36.3)$ \\
Grade $2(\%)$ & $5(22.7)$ & $27(6.4)$ \\
Grade $3(\%)$ & $2(9.1)$ & $60(14.3)$ \\
Grade 2+3 (\%) & $7(31.8)^{*}$ & \\
Small-vessel disease, $n(\%)$ & $11(50)^{*}$ &
\end{tabular}

MBs were located in the basal ganglia in 12 subjects, the cerebellum in four and in the lobes in nine. Two subjects had MBs in both basal ganglia and lobar lesions. Risk profiles of subjects divided into two groups based on location, namely strictly lobar lesions $(n=7)$ and basal ganglia and infratentorial lesions $(n=15)$, were compared. There were no significant difference in baPWV $(1869 \pm 337$ vs. $1791 \pm 299 \mathrm{~cm} / \mathrm{s}$, respectively, $P=0.12)$, carotid IMT $(0.81 \pm 0.31$ vs. $0.87 \pm 0.15 \mathrm{~mm}$, respectively, $P=0.34)$ and FSRS $(8.3 \pm 4.3$ vs. $14.4 \pm 9.7 \%$, respectively, $P=0.11$ ) between subjects with strictly lobar MBs lesions and subjects with MBs located in basal ganglia and infratentorial regions.

\section{Pulse wave velocity and MBs}

To further investigate whether atherosclerotic parameters could predict the presence of $\mathrm{MBs}$, the prevalence of MBs was analyzed by tertile of the respective index (Figure 1). Prevalence showed a highly significant difference among tertiles of baPWV, but was not significantly related to tertile of carotid IMT or FSRS.

To obtain baPWV cutoff values, ROC curve analysis for the presence of MBs was performed. Highest sensitivity-(1-specificity) was obtained with baPWV at $1500 \mathrm{~cm} / \mathrm{s}$, and this value was accordingly defined as high baPWV associated with the presence of MBs. Odds ratios of high baPWV for the presence of MBs are summarized in Table 4. Even after adjustment for other possible confounding parameters, including age, sex, BMI, SBP or PP, LDL-chol, HDL-chol, TG, HbAlc, hypertension and type 2 diabetes, baPWV $\geqslant 1500 \mathrm{~cm} / \mathrm{s}$ was significantly related to the presence of MBs. However, baPWV as a continuous variable was not associated with the presence of MBs in any models examined. High baPWV $(\geqslant 1500 \mathrm{~cm} / \mathrm{s})$ was also significantly related to the presence of MBs with $\operatorname{SVD}\left(\chi^{2}=10.5, P=0.001\right)$, but not to the presence of those without SVD. High baPWV was also related to the presence of MBs located in the basal ganglia/infratentorial region (odds ratio $6.7\left(\chi^{2}=3.9, P=0.049\right)$ ), as well as MBs strictly located in the lobes $\left(\chi^{2}=7.7, P=0.006\right)$.

\section{DISCUSSION}

In this study, we found that older age and hypertension were risk factors for MBs in studied subjects. We further found that MBs were associated with advanced risk factors for stroke, indexed by FSRS, which predicts the risk of stroke in the next 10 years. ${ }^{21}$ These findings confirm previous findings that older age and hypertension are risk factors for MBs. ${ }^{2-4} \mathrm{To}$ our knowledge, this is the first report to demonstrate a highly significant association between MBs and arterial stiffness. 

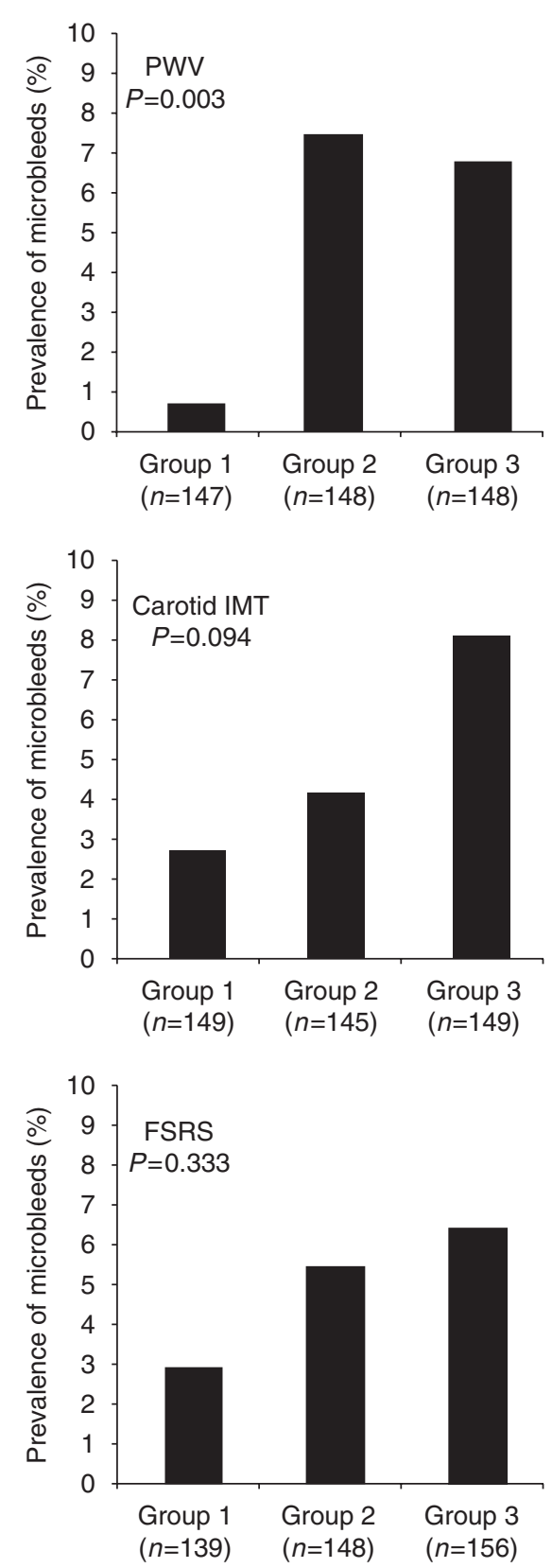

Figure 1 Prevalence of MBs by tertiles of atherosclerotic parameters. $P$-values obtained by the $\chi^{2}$-test are shown. baPWV, brachial-ankle pulse wave velocity; carotid IMT, carotid intima-media thickness; FSRS, Framingham stroke risk score; MBs, microbleeds.

MBs are often incidentally identified in patients with stroke. ${ }^{2-4}$ Although prevalence in these patients varies widely among studies, a recent meta-analysis reported an incidence of $33.5-40 \%$ in ischemic stroke and $60.4-68 \%$ in intracerebral hemorrhage. ${ }^{2-4}$ In contrast, incidence in the general population has been reported at $5 \%$, ranging from 3.7 to $8.5 \% .^{2-4}$ Using a custom three dimensional T2* sequence on a $1.5 \mathrm{~T}$ MRI, which detects MBs with nearly two times the sensitivity of conventional two-dimensional $\mathrm{T}^{*}$ imaging; however, the Rotterdam study recently reported a much higher prevalence in the general population of $23.5 \% .{ }^{10}$ In the present study, using 3T MRI, which in turn has greater sensitivity than $1.5 \mathrm{~T} \mathrm{MRI},{ }^{22}$ we observed a prevalence of $5 \%$ in our studied population free from a history of stroke. These differences in prevalence might be explained by differ- ences in the clinical background of the study populations, particularly with regard to the ratio of hypertensive patients, which was $71.9 \%$ in the Rotterdam study compared with $56.2 \%$ in the present study. ${ }^{10}$ Findings that the prevalence of MBs is higher in hypertensive patients may thus explain this difference, at least in part. $^{23}$

Stiffening of an artery is a major pathological manifestation of the senescence of the artery, particularly with regard to the aorta. ${ }^{24}$ The major underlying mechanism of stiffness is the fatigue and eventual rupture of the elastic fibers by repetitious cyclic stress. ${ }^{24} \mathrm{PWV}$ is a clinical parameter of the stiffness of the artery, which between the brachium and ankle represents not only stiffness of the aorta but also of the peripheral brachial and iliac arteries. ${ }^{20}$ Given that older age and hypertension are two major independent risk factors for arterial stiffness, the mechanisms underlying stiffness and MBs may be the same. ${ }^{24}$

Arterial stiffness has been associated with numerous risk factors for stroke and is an independent risk factor for cardiovascular diseases, including stroke. ${ }^{25}$ Its prognostic power for cardiovascular events has been prospectively demonstrated. ${ }^{26}$ In our study, we found that baPWV $\geqslant 1500 \mathrm{~cm} / \mathrm{s}$ was an independent predictor of the presence of MBs. This significant association between high baPWV and MBs remained after correction for confounding factors, including BP parameters and age.

Our finding that MBs were closely associated with SVD, which is manifested by lacunar infarcts and advanced deep white matter lesions, is consistent with previous findings. Although the mechanism underlying the link between arterial stiffness and MBs has not been elucidated, one recent hypothesis states that aortic stiffness may lead to brain damage by inducing changes in the microcirculation. ${ }^{24}$ Stiffening may prevent the cushioning of flow pulsations created by LV ejection: in this scenario, the higher resting blood flow causes the dilation of blood vessels leading down to the brain's capillary circulation, which in turn facilitates the transmission of pulsations into the smallest vessels in the brain. Interestingly, arterial pulsatility in dementia is greater in those patients with leukoaraiosis than in those without, ${ }^{27}$ and an increase in arterial stiffness is associated with the presence of $\mathrm{PVH}^{28}$

In postmortem pathological examination, it has been shown that MBs are the result of leakage of blood from damaged arteriolar walls. ${ }^{29}$ The pathology in vessels affected by MBs is most often lipofibrohyalinosis, characterized by destruction of normal vascular wall architecture in small vessels. ${ }^{29}$ These changes can also cause ischemic events by in situ thrombosis or reduced flow distal to the lesion. ${ }^{29}$ On the other hand, abnormal permeability of the arteriolar blood-brain barrier has been suggested to lead to the extravasation of blood components as a common feature of MBs associated with SVD. ${ }^{2,30}$ Based on these findings with present results, it is also conceivable that high pulsatility load could further deteriorate and damage the small vessel wall to cause leakage of blood.

On the other hand, we also observed MBs in the absence of SVD. It is noteworthy that risk factor profiles significantly differed between MBs subjects with and without SVD. FSRS was significantly higher in MBs subjects with SVD, but did not differ from the control in those without SVD. Indeed, these latter subjects showed no difference from the control in any parameter, including baPWV. These findings suggest that the pathogenesis of MBs may differ between MBs with and without SVD.

The etiological background of MBs differs in accordance with their location: ${ }^{2-4,10,31}$ those in the basal ganglia, brainstem and cerebellum are associated with SVD and risk factors including hypertension, whereas those restricted to the lobar, cortical and subcortical regions show no association with risk factors and are thought to be consequent to CAA. ${ }^{2-4,10,31}$ In the present study, there was no significant difference 
Table 4 Logistic regression analyses for the presence of microbleeds

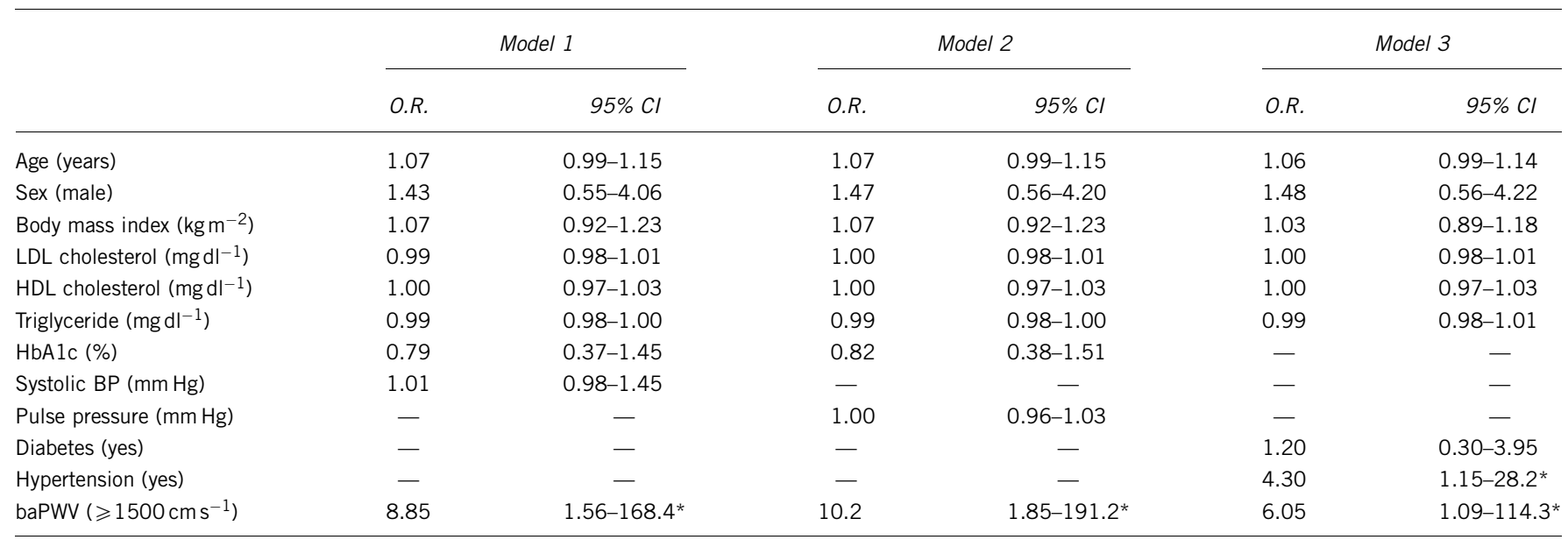

Model 1: corrected for age, sex, BMI, SBP, LDL-C, HDL-C, TG, HbA1c and systolic BP.

Model 2: corrected for age, sex, BMI, SBP, LDL-C, HDL-C, TG, HbAlc and pulse pressure.

Model 3: corrected for age, sex, BMI, LDL-C, HDL-C, TG, diabetes and hypertension.

$\mathrm{Cl}$ : Wald confidence interval for adjusted odds ratio.

${ }^{*} P<0.05$.

in baPWV between subjects with MBs solely located in the lobe and those with MBs in the basal ganglia and infratentorial regions.

In a series of 2060 postmortem examinations, 19 of 59 (32\%) cases of non-CAA-related intracerebral hemorrhage were found in the lobes, whereas 11 of $56(20 \%)$ cases of CAA-related hemorrhage were located in the basal ganglia and brainstem. ${ }^{32} \mathrm{~A}$ second postmortem study in hypertensive patients similarly demonstrated that CAA was associated with not only lobar but also deep brain hemorrhage. ${ }^{33}$ These findings indicate that CAA may also play a role in hemorrhage in patients with more evident risk factors. The structural changes in the vascular wall related to CAA are associated with fibrinoid necrosis, focal vessel wall fragmentation and microaneurysms, which all predispose to blood vessel leakage. ${ }^{31}$ These findings may suggest that pathological alterations associated with arterial stiffness, including an increase in pulsatile stimuli, may also underlay the association between MBs and CAA.

Lee et al. ${ }^{11}$ demonstrated that left ventricular hypertrophy was an independent risk factor for the severity of MBs. We also observed that LVMI was an independent predictor for the presence of silent lacunar infarct. ${ }^{34}$ In the present study, however, LVH defined by ECG was not significantly different between subjects with and without MBs. As several studies have reported ECG-defined LVH is not reliable, ${ }^{35,36}$ which may explain the failure of the association between LVH and MBs in the present study.

Importantly, the question of whether the occurrence of MBs in a general population is a risk for future symptomatic intracerebral hemorrhage remains unanswered. Although several prospective studies have demonstrated that the presence or number of MBs is significantly related to the development of intracerebral hemorrhage in patients with leukoaraiosis and stroke as well as moya-moya disease, ${ }^{6,37-39}$ a recent multicenter study demonstrated thrombolysis did not increase the risk of intracerebral hemorrhage in stroke patients with MBs. ${ }^{40}$ Although recent guidelines recommend the use of aspirin for the primary prevention of cardiovascular diseases in subjects with high-risk profiles, there is concern that this may facilitate intracerebral hemorrhage in those with MBs. ${ }^{41}$ A meta-analysis of primary prevention with a total of 95456 individuals demonstrated that aspirin increased the risk of intracerebral hemorrhage 1.69 times in men. ${ }^{42}$ However, the absolute rate was low, at only 50 cases among 17960 male aspirin users; assuming $5 \%$ of them had MBs, these findings may indicate that the absolute rate of developing symptomatic intracerebral hemorrhage in subjects with MBs would not be high enough to justify the withdrawal of aspirin and subsequent loss of benefit. Nevertheless, the natural history and possible adverse effects of antiplatelet and anticoagulation therapy in subjects with MBs among the general population clearly requires elucidation.

In hypertensive patients with stroke, calcium channel blockers, ACE inhibitors or angiotensin II receptor blockers have been proposed as compelling antihypertensive agents. ${ }^{43}$ Furthermore, these classes of antihypertensive drugs have been shown to reduce arterial stiffness ${ }^{44}$ and reserve cerebral blood flow, thus are thought to be suitable for patients with SVD. ${ }^{45}$ These findings may suggest that the classes of antihypertensive drugs may influence on the development of SVD including MBs. In the preset study, ACE inhibitors were more frequently used in patients with MBs than those without MBs. $\beta$-blockers, which have been shown to be less effective in stroke prevention, ${ }^{46}$ were not used in hypertensive patients with MBs. These findings indicate that classes of antihypertensive drugs were rather properly selected in patients with MBs. However, it would be necessary to investigate whether antihypertensive agents facilitates the progression of MBs in prospective studies.

We need to be aware of several limitations of the study. Participants in the study voluntarily applied for the medical check-up at the AntiAging Center. It is conceivable that participants had high levels of health consciousness, and may differ from the general population. Accordingly, it should be cautious to apply the present findings to the general population. Furthermore, the cross-sectional nature of the study does not allow us to determine the time relationship between high baPWV and MBs. A longitudinal observational study would be necessary to clarify a causal role of arterial stiffness on the development of MBs.

In summary, our analysis using $3 \mathrm{~T}$ high resolution MRI showed a prevalence of MBs in apparently healthy Japanese subjects of $5 \%$. The etiology of this condition appears heterogenous, however, although their presence was significantly associated with arterial stiffness and stroke risk score, background risk profile and degree of arterial stiffness differed significantly between MBs subjects with and without SVD. The association with high baPWV remained irrespective of MBs location, whether strictly located in the lobes or in the basal ganglia 
and infratentorial regions. Assessment of arterial stiffness may be useful in identifying subjects at high risk of the occurrence of MBs.

\section{CONFLICT OF INTEREST}

The authors state no conflict of interest.

\section{ACKNOWLEDGEMENTS}

This study was supported by grants-in-aid for Scientific Research from The Ministry of Education, Culture, Sports, Science and Technology of Japan; The Ministry of Health, Labour and Welfare of Japan; the Japan Arteriosclerosis Prevention Fund; a grant-in-aid from Mitsubishi Pharma Research Foundation; and a Research Promotion Award from Ehime University.

1 Offenbacher H, Fazekas F, Schmidt R, Koch M, Fazekas G, Kapeller P. MR of cerebral abnormalities concomitant with primary intracerebral hematomas. Am J Neuroradiol 1996; 17: 573-578.

2 Koennecke HC. Cerebral microbleeds on MRI: prevalence, associations, and potential clinical implications. Neurology 2006; 66: 165-171.

3 Cordonnier C, Al-Shahi Salman R, Wardlaw J. Spontaneous brain microbleeds: systematic review, subgroup analyses and standards for study design and reporting. Brain 2007: 130: 1988-2003.

4 Viswanathan A, Chabriat H. Cerebral microhemorrhage. Stroke 2006; 37: 550-555.

5 Naka H, Nomura E, Wakabayashi S, Kajikawa H, Kohriyama T, Mimori Y, Nakamura S, Matsumoto M. Frequency of asymptomatic microbleeds on T2*-weighted MR images of patients with recurrent stroke: association with combination of stroke subtypes and leukoaraiosis. AJNR Am J Neuroradiol 2004; 25: 714-719.

6 Naka H, Nomura E, Takahashi T, Wakabayashi S, Mimori Y, Kajikawa H, Kohriyama T, Matsumoto M. Combinations of the presence or absence of cerebral microbleeds and advanced white matter hyperintensity as predictors of subsequent stroke types. AJNR Am J Neuroradiol 2006; 27: 830-835.

7 Imaizumi T, Horita Y, Hashimoto Y, Niwa J. Dotlike hemosiderin spots on T2*-weighted magnetic resonance imaging as a predictor of stroke recurrence: a prospective study. J Neurosurg 2004; 101: 915-920.

8 Kidwell CS, Saver JL, Villablanca JP, Duckwiler G, Fredieu A, Gough K, Leary MC, Starkman S, Gobin YP, Jahan R, Vespa P, Liebeskind DS, Alger JR, Vinuela F. Magnetic resonance imaging detection of microbleeds before thrombolysis: an emerging application. Stroke 2002; 33: 95-98.

9 Wong KS, Chan YL, Liu JY, Gao S, Lam WW. Asymptomatic microbleeds as a risk factor for aspirin-associated intracerebral hemorrhages. Neurology 2003; 60: 511-513.

10 Vernooij MW, van der Lugt A, Ikram MA, Wielopolski PA, Niessen WJ, Hofman A, Krestin GP, Breteler MM. Prevalence and risk factors of cerebral microbleeds: the Rotterdam Scan Study. Neurology 2008; 70: 1208-1214.

11 Lee SH, Park JM, Kwon SJ, Kim H, Kim YH, Roh JK, Yoon BW. Left ventricular hypertrophy is associated with cerebral microbleeds in hypertensive patients. Neurology 2004; 63: 16-21.

12 Cullen KM, Kocsi Z, Stone J. Microvascular pathology in the aging human brain: evidence that senile plaques are sites of microhemorrhages. Neurobiol Aging 2006; 27: 1786-1796.

13 Hanon O, Haulon S, Lenoir H, Seux ML, Rigaud AS, Safar M, Girerd X, Forette F. Relationship between arterial stiffness and cognitive function in elderly subjects with complaints of memory loss. Stroke 2005; 36: 2193-2197.

14 O'Rourke MF, Safar ME. Relationship between aortic stiffening and microvascular disease in brain and kidney: cause and logic of therapy. Hypertension 2005; 46: 200-204.

15 Fazekas F, Chawluk JB, Alavi A, Hurtig HI, Zimmerman RA. MR signal abnormalities at $1.5 \mathrm{~T}$ in Alzheimer's dementia and normal aging. Am J Roentgenol 1987; 149: 351-356.

16 Uehara T, Tabuchi M, Mori E. Risk factors for silent cerebral infarcts in subcortical white matter and basal ganglia. Stroke 1999; 30: 378-382.

17 Japanese Society for Detection of Asymptomatic Brain Disease. A new guideline making committee of a brain dock. Braindock Guideline 2003, 25-28, 39-44.

18 Kurata M, Okura T, Watanabe S, Higaki J. Association between carotid hemodynamics and asymptomatic white and gray matter lesions in patients with essential hypertension. Hypertens Res 2005; 28: 797-803.

19 Jiang Y, Kohara K, Hiwada K. Low wall shear stress in carotid arteries in subjects with left ventricular hypertrophy. Am J Hypertens 2000; 13: 892-898.

20 Yamashina A, Tomiyama H, Takeda K, Tsuda H, Arai T, Hirose K, Koji Y, Hori S, Yamamoto $Y$. Validity, reproducibility, and clinical significance of noninvasive brachialankle pulse wave velocity measurement. Hypertens Res 2002; 25: 359-364.

21 D'Agostino RB, Wolf PA, Belanger AJ, Kannel WB. Stroke risk profile: adjustment for antihypertensive medication. The Framingham Study. Stroke 1994; 25: 40-43.

22 Scheid R, Ott DV, Roth H, Schroeter ML, von Cramon DY. Comparative magnetic resonance imaging at 1.5 and 3 Tesla for the evaluation of traumatic microbleeds. J Neurotrauma 2007; 24: 1811-1816.
23 Lee SH, Bae HJ, Ko SB, Kim H, Yoon BW, Roh JK. Comparative analysis of the spatial distribution and severity of cerebral microbleeds and old lacunes. J Neurol Neurosurg Psychiatry 2004; 75: 423-427.

24 O'Rourke MF, Hashimoto J. Mechanical factors in arterial aging: a clinical perspective. J Am Coll Cardiol 2007; 50: 1-13.

25 Laurent S, Boutouyrie P. Arterial stiffness and stroke in hypertension: therapeutic implications for stroke prevention. Cns Drugs 2005; 19: 1-11.

26 Meaume S, Benetos A, Henry OF, Rudnichi A, Safar ME. Aortic pulse wave velocity predicts cardiovascular mortality in subjects $>70$ years of age. Arterioscler Thromb Vasc Biol 2001; 21: 2046-2050.

27 Bateman GA. Pulse-wave encephalopathy: a comparative study of the hydrodynamics of leukoaraiosis and normal-pressure hydrocephalus. Neuroradiology 2002; 44: 740-748.

28 Ohmine T, Miwa Y, Yao H, Yuzuriha T, Takashima Y, Uchino A, Takahashi-Yanaga F, Morimoto S, Maehara Y, Sasaguri T. Association between arterial stiffness and cerebral white matter lesions in community-dwelling elderly subjects. Hypertens Res 2008; 31 : 75-81.

29 Werring DJ, Coward LJ, Losseff NA, Jäger HR, Brown MM. Cerebral microbleeds are common in ischemic stroke but rare in TIA. Neurology 2005; 65: 1914-1918.

30 Wardlaw JM, Lewis SC, Keir SL, Dennis MS, Shenkin S. Cerebral microbleeds are associated with lacunar stroke defined clinically and radiologically, independently of white matter lesions. Stroke 2006; 37: 2633-2636.

31 Chao CP, Kotsenas AL, Broderick DF. Cerebral amyloid angiopathy: CT and MR imaging findings. Radiographics 2006; 26: 1517-1531.

32 Attems J, Lauda F, Jellinger KA. Unexpectedly low prevalence of intracerebral hemorrhages in sporadic cerebral amyloid angiopathy: an autopsy study. J Neurol 2008; 255: 70-76.

33 Ritter MA, Droste DW, Hegedüs K, Szepesi R, Nabavi DG, Csiba L, Ringelstein EB. Role of cerebral amyloid angiopathy in intracerebral hemorrhage in hypertensive patients. Neurology 2005; 64: 1233-1237.

34 Kohara K, Zhao B, Jiang Y, Takata Y, Fukuoka T, Igase M, Miki T, Hiwada K. Relation of left ventricular hypertrophy and geometry to asymptomatic cerebrovascular damage in essential hypertension. Am J Cardiol 1999; 83: 367-370.

35 Casiglia E, Maniati G, Daskalakis C, Colangeli G, Tramontin P, Ginocchio G, Spolaore P. Left-ventricular hypertrophy in the elderly: unreliability of ECG criteria in 477 subjects aged 65 years or more. The CArdiovascular STudy in the ELderly (CASTEL). Cardiology 1996; 87: 429-435.

36 Ang DS, Ti LL, Struthers AD. The electrocardiogram is an unreliable method of identifying left ventricular hypertrophy in stable, treated angina patients. J Hum Hypertens 2008; 22: 394-400.

37 Jeon SB, Kang DW, Cho AH, Lee EM, Choi CG, Kwon SU, Kim JS. Initial microbleeds at MR imaging can predict recurrent intracerebral hemorrhage. J Neurol 2007; 254: 508-512.

38 Kikuta K, Takagi Y, Nozaki K, Sawamoto N, Fukuyama H, Hashimoto N. The presence of multiple microbleeds as a predictor of subsequent cerebral hemorrhage in patients with moyamoya disease. Neurosurgery 2008; 62: 104-111.

$39 \mathrm{Lee} \mathrm{SH}$, Heo JH, Yoon BW. Effects of microbleeds on hemorrhage development in leukoaraiosis patients. Hypertens Res 2005; 28: 895-899.

40 Fiehler J, Albers GW, Boulanger JM, Derex L, Gass A, Hjort N, Kim JS, Liebeskind DS, Neumann-Haefelin T, Pedraza S, Rother J, Rothwell P, Rovira A, Schellinger PD, Trenkler J, MR STROKE Group. Bleeding risk analysis in stroke imaging before thromboLysis (BRASIL): pooled analysis of $\mathrm{T} 2^{*}$-weighted magnetic resonance imaging data from 570 patients. Stroke 2007; 38: 2738-2744.

41 Mancia G, De Backer G, Dominiczak A, Cifkova R, Fagard R, Germano G, Grassi G, Heagerty AM, Kjeldsen SE, Laurent S, Narkiewicz K, Ruilope L, Rynkiewicz A, Schmieder RE, Struijker Boudier HA, Zanchetti A, Vahanian A, Camm J, De Caterina R, Dean V, Dickstein K, Filippatos G, Funck-Brentano C, Hellemans I, Kristensen SD, McGregor K, Sechtem U, Silber S, Tendera M, Widimsky P, Zamorano JL, Kjeldsen SE, Erdine S, Narkiewicz K, Kiowski W, Agabiti-Rosei E, Ambrosioni E, Cifkova R, Dominiczak A, Fagard R, Heagerty AM, Laurent S, Lindholm LH, Mancia G, Manolis A, Nilsson PM, Redon J, Schmieder RE, Struijker-Boudier HA, Viigimaa M, Filippatos G, Adamopoulos S, Agabiti-Rosei E, Ambrosioni E, Bertomeu V, Clement D, Erdine S, Farsang C, Gaita D, Kiowski W, Lip G, Mallion JM, Manolis AJ, Nilsson PM, O'Brien E, Ponikowski P, Redon J, Ruschitzka F, Tamargo J, van Zwieten P, Viigimaa M, Waeber B, Williams B, Zamorano JL, The task force for the management of arterial hypertension of the European Society of Hypertension. The task force for the management of arterial hypertension of the European Society of Cardiology. 2007 Guidelines for the management of arterial hypertension: the Task Force for the Management of Arterial Hypertension of the European Society of Hypertension (ESH) and of the European Society of Cardiology (ESC). Eur Heart J 2007; 28: 1508-1509.

42 Berger JS, Roncaglioni MC, Avanzini F, Pangrazzi I, Tognoni G, Brown DL. Aspirin for the primary prevention of cardiovascular events in women and men: a sex-specific meta-analysis of randomized controlled trials. JAMA 2006; 295: 306-313.

43 The Japanese Society of Hypertension Guidelines for the Management of Hypertension (JSH 2009). Hypertens Res 2009; 32: 3-107.

44 Takami T, Shigemasa M. Efficacy of various antihypertensive agents as evaluated by indices of vascular stiffness in elderly hypertensive patients. Hypertens Res 2003; 26: 609-614.

45 Kario K, Ishikawa J, Hoshide S, Matsui Y, Morinari M, Eguchi K, Ishikawa S, Shimada K. Diabetic brain damage in hypertension: role of renin-angiotensin system. Hypertension 2005; 45: 887-893.

46 Messerli FH, Grossman E, Goldbourt U. $\beta$-blockers efficacious as first-line therapy for hypertension in the elderly? A systematic review. JAMA 1998; 279: 1903-1907. 